\title{
Exploring the interactions between serum free fatty acids and fecal microbiota in obesity through a machine learning algorithm
}

\author{
Tania Fernández-Navarro ${ }^{\mathrm{a}, ~ e}$, Irene Díaz $^{\mathrm{c}}$, Isabel Gutiérrez-Díaz ${ }^{\mathrm{a}, \text { e }}$, Javier Rodríguez-Carrio ${ }^{\mathrm{b}, \mathrm{f}}$, Ana Suárez ${ }^{\mathrm{b}, \mathrm{f}}$, \\ Clara G. de los Reyes-Gavilán ${ }^{\mathrm{d}, ~ e}$, Miguel Gueimonde ${ }^{\mathrm{d}, ~ e}$, Nuria Salazar ${ }^{\mathrm{d}, \text { e, *, Sonia González }}$, e, * \\ ${ }^{a}$ Area of Physiology, Department of Functional Biology, Faculty of Medicine, University of Oviedo, C/Julián Clavería s/n, 33006 Oviedo, Asturias, Spain \\ ${ }^{\mathrm{b}}$ Area of Immunology, Department of Functional Biology, Faculty of Medicine, University of Oviedo, C/Julián Clavería s/n, 33006 Oviedo, Asturias, Spain \\ ${ }^{c}$ Area of Computer Science and Artificial Intelligence, Department of Computer Science, Faculty of Science, University of Oviedo, C/Federico García Lorca, 18, 33007 Oviedo, Asturias, Spain \\ d Department of Microbiology and Biochemistry of Dairy Products, Instituto de Productos Lácteos de Asturias, Consejo Superior de Investigaciones Científicas (IPLA-CSIC), Paseo Río Linares s/n, \\ 33300 Villaviciosa, Asturias, Spain \\ e Group Diet, Microbiota and Health, Instituto de Investigación Sanitaria del Principado de Asturias (ISPA), Avda. Roma s/n, 33011 Oviedo, Asturias, Spain \\ ${ }^{\mathrm{f}}$ Group Basic and Translational Research in Inflammatory Diseases, Instituto de Investigación Sanitaria del Principado de Asturias (ISPA), Avda. Roma s/n, 33011 Oviedo, Asturias, Spain
}

\section{A R T I C L E IN F O}

\section{Keywords:}

Free fatty acids

Diet

Microbiota

Obesity

EPA

Bacteroides

Bifidobacterium

\begin{abstract}
A B S T R A C T
Serum free fatty acids (FFA) are generally elevated in obesity. The gut microbiota is involved in the host energy metabolism through the regulation of body fat storage, and a link between diet, FFA and the intestinal microbiota seems to exist. Our aim was to explore the interaction among serum FFA levels, gut microbiota, diet and obesity through a model regression tree in 66 subjects (age $52.7 \pm 11.2 \mathrm{y}$ ) classified according to Body Mass Index (BMI). Total and individual FFA were analyzed by colorimetric enzymatic assay and methyl-tert-butylether-based extraction protocol (MTBE), respectively. Microbiota was determined by qPCR and diet through a food frequency questionnaire. Statistical analyses were performed, and predictive factors for obesity were obtained via classification by decision trees using machine learning methods. An obese-linked FFA profile was characterized by decreased eicosapentaenoic (EPA) and increased linoleic, gamma-linolenic and palmitic acids levels simultaneously. Serum EPA and gender were identified as the most significant variables with $100 \%$ and $80 \%$ of importance, respectively. Palmitic acid, Bifidobacterium and Faecalibacterium explained $>30 \%$, followed by Bacteroides group with $20 \%$ and docosahexaenoic acid (DHA) almost with $15 \%$ of importance. Also, the regression tree model obtained for predicting obesity, showed a non-obese-linked profile, independently of gender, with serum EPA $>0.235 \mu \mathrm{g} / \mathrm{mL}$ and Bacteroides $>9.055 \log n^{\circ}$ cells per $\mathrm{g}$ of feces. Moreover, Faecalibacterium and Bifidobacterium seemed to play an important role by complementing the levels of FFA in predicting obesity in males and females, respectively.
\end{abstract}

\section{Introduction}

Obesity has been recognized by the World Health Organization (WHO) as the epidemic of the 21st century due to the alarming increase in its incidence worldwide and its impact on the morbidity and mortality, that threatens to overwhelm the healthcare systems (World Health Organization, 2016). This multifactorial disorder results from the interaction among a plethora of factors, including genetic and environmental ones, with special focus on the Westernized dietary patterns and the sedentary lifestyle (De Los Reyes-Gavilán, Delzenne, González, Gueimonde, \& Salazar, 2014). The long-term excessive caloric intake promotes adipose tissue inflammation (DeMarco, Aroor, \& Sowers, 2014; Emanuela et al., 2012; Trayhurn, 2005), leading to ectopic lipid accumulation (Cavalcante-Silva, Galvão, da Silva, de Sales-Neto, \&
Rodrigues-Mascarenhas, 2015). Despite this understanding of the underlying factors, after more than two decades of research, there is not still a clear conclusion about the role of free fatty acids (FFA) metabolism in obesity. It is generally acknowledged that the concentration of circulating FFA is increased in obesity and that high levels of FFA are implicated in the pathogenesis of obesity-related insulin resistance, type 2 diabetes and cardiovascular diseases (Karpe, Dickmann, \& Frayn, 2011). However, a recent meta-analysis compiling results from 43 studies has reported only normal or moderately increased levels of FFA in obesity (Karpe et al., 2011). Considering that plasma FFA concentrations are mainly produced by the breakdown of intracellular triglycerides into fatty acids, it is reasonable to expect a large degree of variation in the fatty acid profile as depending on the composition of the subject's diet. Despite that both, saturated and polyunsaturated fatty acids, contribute to the increase in FFA (Lee et al., 2006) and pro-

\footnotetext{
* Corresponding authors.

Email address: soniagsolares@uniovi.es (S. González)
} 
vide similar energy content, they could have a different behavior from a metabolic point of view and, as a consequence, a differential role in obesity.

Several authors have proposed that different fatty acids are able to drive different changes in the composition and functionality of the intestinal microbiota, thus contributing to host lipid metabolism (Rodríguez-Carrio et al., 2017), to the development of obesity (Karlsson et al., 2013; Khan, Nieuwdorp, \& Bäckhed, 2014; Le Chatelier et al., 2013; Tremaroli \& Bäckhed, 2012; Zhao, 2013), and to the higher proinflammatory status classically associated to subjects with this pathology (Clarke et al., 2014; Rodríguez-Carrio et al., 2017). Results from intervention studies in animals and humans have reported changes in certain intestinal microbial populations in the context of obesity although, the specific mechanisms that link rearrangements of the gut microbial composition to the pathogenesis of obesity and related metabolic diseases remain mostly unexplored (Dao et al., 2018). Although several authors have found an increase in the ratio Firmicutes/Bacteroidetes, these results remain controversial. Some Bifidobacterium and Lactobacillus species as well as Akkermansia muciniphila have been associated with lean phenotype (Dao et al., 2016; Million et al., 2012). Other microorganisms such as Faecalibacterium prausnitzii showed inconsistent results about its role in obesity (Feng et al., 2014). In this context, the aim of this study was to analyze the role of FFA in obesity and to identify possible serum FFA profile/s associated with this condition, as well as the role of the gut microbiota in this relationship. Since the traditional recommendations based on weight loss through diet modification and exercise have not been successful enough to fight against obesity, the identification of possible serum FFA profiles associated with obesity could open new dietary or pharmacological ways to normalize serum FFA levels and improve the response of the obese people to treatments.

\section{Subjects and methods}

\subsection{Participants}

The study sample comprised 66 adult volunteers, 26 men and 40 women, aged from 19 to 67 years (mean \pm SD, $52.7 \pm 11.2$ ) and with a BMI ranging from 19.0 to $40.0 \mathrm{~kg} / \mathrm{m}^{2}$, that were recruited in Asturias Region (Northern Spain). In a personal interview, volunteers were informed of the objectives of the study and those deciding to participate gave their fully informed written consent. Subjects were initially classified according to their Body Mass Index (BMI) (Salas-Salvado, Rubio, Barbany, \& Moreno, 2007). Inclusion criteria were not being diagnosed of autoimmune diseases, inflammatory bowel disease or other conditions known to affect the intestinal function, as well as not having undergone medical treatment with oral corticoids, immunosuppressive agents, monoclonal antibodies, antibiotics or immunotherapy or not having consumed consciously any supplement containing probiotics or prebiotics during the previous month.

Ethical approval for this study was obtained from the Bioethics Committee of CSIC (Consejo Superior de Investigaciones Científicas) and from the Regional Ethics Committee for Clinical Research (Servicio de Salud del Principado de Asturias $n^{\circ} 13 / 2010$ ) in compliance with the Declaration of Helsinki of 1964. All experiments were carried out in accordance with approved guidelines and regulations.

\subsection{Anthropometric measures}

At the time of carrying out the blood extraction, between eight and nine o'clock in the morning, and after an over-night fast, anthropometric measures were taken. Height was registered using a stadiometer with an accuracy of $\pm 1 \mathrm{~mm}$ (Año-Sayol, Barcelona, Spain). Subjects stood barefoot, in an upright position and with the head positioned in the Frankfort horizontal plane. Weight was measured on a scale with an accuracy of $\pm 100 \mathrm{~g}$ (Seca, Hamburg, Germany). BMI was calculated from the formula: weight $(\mathrm{Kg}) /$ height $\left(\mathrm{m}^{2}\right)$ and stratified according to the Sociedad Española para el Estudio de la Obesidad (SEEDO) (Salas-Salvado et al., 2007) criteria: normal weight $<25.0 \mathrm{~kg} / \mathrm{m}^{2}$, overweight $25.0-26.9 \mathrm{~kg} / \mathrm{m}^{2}$, and pre-obesity/obesity $\geq 27.0 \mathrm{~kg} / \mathrm{m}^{2}$. Body fat percentage was measured by bioelectrical impedance (BIA) with $\pm 1 \%$ variation, with subjects in light clothes and in fasted state (Tanita Corporation of America, Inc., Arlington Heights, IL, USA).

\subsection{Blood Biochemical analyses}

Fasting blood samples were drawn by venipuncture and collected in separate tubes for serum and plasma. Samples were kept on ice and centrifuged (1000 x g, $15 \mathrm{~min}$ ) within $2-4 \mathrm{~h}$ after collection. Plasma and serum aliquots were kept at $-20^{\circ} \mathrm{C}$ until analyses were performed.

Plasma glucose, cholesterol, and triglycerides were determined by standard methods. Serum levels of C-reactive protein (CRP) were determined by using a CRP Human Instant ELISA kit (Ebioscience, San Diego, CA, USA), and those of malondialdehyde (MDA) with a colorimetric assay of lipid peroxidation (Byoxytech LPO-586, Oxis International S.A., Paris, France); the within-run coefficient of variation ranged from $1.2 \%$ to $3.4 \%$, depending on the concentration of MDA (Gerard-Monnier et al., 1998).

Serum leptin was measured by a sensitive ELISA test (Human Leptin ELISA Development Kit, 900-K90 PeproTech Inc., Rocky Hill, NJ, USA) according to the manufacturer's instructions. The detectable concentration range was $63-4000 \mathrm{pg} / \mathrm{mL}$. The intra-assay and inter-assay coefficients of variation were $5.21 \%$ and $5.20 \%$, respectively.

\subsection{Free fatty acids assessment}

Individual FFA were analyzed by liquid chromatography-mass spectrometry following a methyl-tert-butylether (MTBE) extraction protocol as previously described (Pizarro, Arenzana-Rámila, Pérez-Del-Notario, Pérez-Matute, \& González-Sáiz, 2013). Briefly, serum samples were spiked with an internal standard (heptadecanoic acid) and organic phases were extracted in MTBE after protein precipitation with methanol. The extraction was repeated, and the organic phases were collected, dried and re-dissolved in the mobile phase. The levels of FFA in the samples were analyzed in a Dionex Ultimate 3000 HPLC system equipped with a Zorbax Eclipse Plus C18 column and mass detection was performed in a Bruker Impact II q-ToF mass spectrometer with electrospray ionization (negative mode).

Total FFA serum levels were quantified by means of a colorimetric assay using a commercial kit (NEFA kit half-microtest, Roche Life Sciences, Penzberg, Germany) following the protocol from the manufacturer, as previously described (Pizarro et al., 2013).

\subsection{Fecal collection and microbial analysis}

Participants received detailed instructions to collect fecal samples and were provided with a sterile container. Samples were immediately frozen at $-20{ }^{\circ} \mathrm{C}$ after deposition. For analyses, fecal samples were melted, weighed, diluted $1 / 10$ in sterile PBS, and homogenized in a LabBlender 400 Stomacher (Seward Medical, London, UK) for $4 \mathrm{~min}$; the DNA was extracted using the QIAamp DNA stool mini kit (Qiagen, Hilden, Germany) as previously described (Arboleya et al., 2012). Quantification of different bacterial populations that covered the major bacterial groups present in the gut microbial ecosystem (Table 1) was performed in feces with a 7500 Fast Real-Time PCR System (Applied Biosystems, Foster City, CA, USA) using SYBR Green PCR Master Mix (Applied Biosystems) (Arboleya et al., 2012). One microliter of template fecal DNA $(\sim 5 \mathrm{ng})$ and $0.2 \mu \mathrm{M}$ of each primer were added to the $25 \mu \mathrm{L}$ reaction mixture. PCR cycling consisted of an initial cycle of $95^{\circ} \mathrm{C} 10 \mathrm{~min}$, followed by 40 cycles of $95^{\circ} \mathrm{C} 15 \mathrm{~s}$, and $1 \mathrm{~min}$ at the appropriate primer-pair temperature (Table 1 ). The number of cells was determined by comparing the $\mathrm{Ct}$ values obtained from a standard curve 
Table 1

Bacterial groups, standard cultures, primers, and annealing temperatures (Tm) used for qPCR in this study.

\begin{tabular}{|c|c|c|c|}
\hline Microbial target & Strain used for standard curve & Primer sequence $5^{\prime}-3^{\prime}$ & $\operatorname{Tm}\left({ }^{\circ} \mathrm{C}\right)$ \\
\hline \multirow[t]{2}{*}{ Akkermansia } & Akkermansia muciniphila & F: CAGCACGTGAAGGTGGGGAC & 60 \\
\hline & CIP 107961 & R: CCTTGCGGTTGGCTTCAGAT & \\
\hline Bacteroides group & Bacteroides thetaiotaomicron & F: GAGAGGAAGGTCCCCCAC & 60 \\
\hline Bacteroides-Prevotella-Porphyromonas & DSMZ 2079 & R: CGCKACTTGGCTGGTTCAG & \\
\hline \multirow[t]{2}{*}{ Bifidobacterium } & Bifidobacterium longum & F:GATTCTGGCTCAGGATGAACGC & 60 \\
\hline & NCIMB 8809 & R: CTGATAGGACGCGACCCCAT & \\
\hline \multirow[t]{2}{*}{ Faecalibacterium } & Faecalibacterium prausnitzii & F:GGAGGAAGAAGGTCTTCGG & 60 \\
\hline & DSMZ 17677 & R: AATTCCGCCTACCTCTGCACT & \\
\hline Clostridia XIVa group & Blautia coccoides & F: CGGTACCTGACTAAGAAGC & 55 \\
\hline Blautia coccoides-Eubacterium rectale & DSMZ 935 & R: AGTTTYATTCTTGCGAACG & \\
\hline \multirow[t]{2}{*}{ Lactobacillus group } & Lactobacillus gasseri & F: AGCAGTAGGGAATCTTCCA & 60 \\
\hline & IPLA IF7/5 & R: CATGGAGTTCCACTGTCCTC & \\
\hline
\end{tabular}

This table is adapted from Arboleya et al., 2012

constructed using the pure cultures of appropriate strains that were grown overnight in GAM (Gifu Anaerobic Medium) (Nissui Pharmaceutical Co., Tokyo, Japan) under anaerobic conditions (Table 1). The Ct values were plotted as a linear function of the base-10 logarithm of the number of cells calculated by plate counting. Fecal DNA extracts were analyzed and the mean quantity per gram of fecal wet weight was calculated.

\subsection{Nutritional assessment}

Dietary intake was assessed in a personal interview by means of an annual semi-quantitative food frequency questionnaire (FFQ) method which details 160 items and that has been widely used and validated in previous studies (Cuervo et al., 2014, 2015). The consumption of foods was converted into energy and macronutrients using the food composition tables developed by the Centro de Enseñanza Superior de Nutrición y Dietética (CESNID) (CESNID, 2008).

\subsection{Data analysis}

Statistical analysis was performed using the IBM SPSS program version 22.0 (IBM SPSS, Inc., Chicago, IL, USA). Goodness of fit to the normal distribution was analyzed by means of the Kolmogorov-Smirnov test. When the distribution of variables was skewed, the natural logarithm of each value was used in the statistical test. Overall, categorical variables were summarized with percentages while continuous variables were summarized using means and standard deviations. The chi-squared test and independent samples $t$-test were used for group comparisons where appropriate. Differences in general characteristics, anthropometric, life-style and blood parameters, as well as total and main individual FFA were assessed in accordance to BMI classification by means of U-Mann Whitney test, and were calculated using normal weight volunteers as reference. Also, lineal regression analyses, adjusted by gender, were used to investigate the association between serum FFA in obesity with BMI, percentage of body fat and serum leptin as adiposity factors, and with serum MDA and CRP as lipid peroxidation and inflammation biomarkers, respectively. The statistical parameters employed were $\beta$ (standardized regression coefficient) and $R^{2}$ (coefficient of multiple determinations). The conventional probability value for significance (0.05) was used in the interpretation of results.

In addition, predictive factors for obesity were obtained via machine learning techniques. In particular, different methods were tested, such as decision tree based (C5.0), recursive partitioning, ensemble methods or support vector machines (Kuhn \& Johnson, 2016). The performance of these methods is estimated through resampling techniques. Most resampling techniques operate similarly: a subset of samples is used to fit a model and the remaining samples are used to estimate the efficacy of the model. This process is repeated multiple times and the results are aggregated and summarized. In particular, k-cross validation randomly partitioned into $\mathrm{k}$ sets of roughly equal size. A model is fit using all samples except the first subset (called the first fold). The held-out samples are predicted by this model and used to estimate performance measures. The first subset is returned to the training set and the procedure repeats with the second subset held out, and so on. The $\mathrm{k}$ resampled estimates of performance are summarized and used to select the best model. In this work, $\mathrm{k}$ was set to 10 . On the other hand, all the methods were optimized according to their parameters with regard to the usual evaluation metrics for this kind of problems. These metrics are Sensitivity, Specificity (Powers, 2011). Given an obesity group, normal weight $<25.0 \mathrm{~kg} / \mathrm{m}^{2}$, overweight $25.0-26.9 \mathrm{~kg}$ / $\mathrm{m}^{2}$, and pre-obesity/obesity $27.0-40.0 \mathrm{~kg} / \mathrm{m}^{2}$, these metrics evaluate the performance of a method in predicting each group. In this sense, Sensitivity measures the proportion of individuals belonging to a certain obesity class correctly identified as such (for example, the proportion of normal weight individuals correctly classified by the method as normal weights). Specificity measures the proportion of individuals not belonging to a certain obesity class correctly identified. The overall Specificity and Sensitivity is obtained by averaging over all the classes. The experiments were performed using $\mathrm{R}$ package version 3.4.3, in particular $\mathrm{R}$ Weka package version 0.4 .36.

The best method to build the classification model was selected considering both interpretability and performance in terms of Specificity and Sensitivity. Interpretability is an important issue in this field. For this reason, three of the four selected methods (decision trees, recursive partitioning and ensemble methods) are easy to interpret, at least, easier than support vector machines, that are less interpretable but, more efficient and accurate in general. Recursive partitioning is not able to correctly identify individuals belonging to a certain obesity, which leads to a null Sensitivity and thus, it is not considered anymore. Ensemble method reached a performance close to that obtained by C5.0. In fact, the averaged Sensitivity was $0.64 \pm 0.10$ and the Specificity was $0.86 \pm 0.06$. On the other hand, the averaged Sensitivity obtained by support vector machines was $0.57 \pm 0.16$ and the Specificity was $0.83 \pm 0.54$. Regarding C5.0, its averaged Sensitivity and Specificity were respectively $0.71 \pm 0.09$ and $0.86 \pm 0.05$. Note that C5.0 reached the higher performance considering both Specificity and Sensitivity. In addition, it is more interpretable than the other methods. Thus, considering both parameters, C5.0 was selected to build the classification model. C5.0 performance, according to BMI, is shown in Table 2.

Table 2

Sensitivity and Specificity according to BMI $\left(\mathrm{kg} / \mathrm{m}^{2}\right)$.

\begin{tabular}{|c|c|c|c|}
\hline & Normal weight & Over weight & Pre-obesity/Obesity \\
\hline & $<25.0$ & $25.0-26.9$ & $\geq 27.0$ \\
\hline & $\mathrm{N}=20$ & $\mathrm{~N}=12$ & $\mathrm{~N}=34$ \\
\hline Sensitivity & $0.68 \pm 0.05$ & $0.59 \pm 0.15$ & $0.87 \pm 0.08$ \\
\hline Specificity & $0.86 \pm 0.05$ & $0.97 \pm 0.03$ & $0.76 \pm 0.08$ \\
\hline
\end{tabular}


C5.0 builds a decision tree as classification model. The strategy to construct the tree is based on a divide and conquers strategy. The first node in the tree is called the root. A node with outgoing edges is called test node and a node without outgoing edges is a leaf. Each path from the root of the tree to a leaf determines a region, that is, a more homogeneous group subset of the input data. Initially, the whole training set is associated with a leaf. Applying a recursive procedure, it is decided using a test if the set associated to a leaf is split into smaller subsets associated to new leaves. When a subset is homogeneous (in some sense) the procedure halts and the node is labeled as a leaf (terminal node). In particular, C5.0 selects the split that maximizes Gain Ratio. As it was previously noted, C5.0 was optimized according to bootstrapping trials, winnowing and model output (Kuhn \& Johnson, 2016), obtaining that the best configuration was 1 bootstrapping trial, no winnowing and tree model as output.

\section{Results}

The general characteristics of the sample, classified according to BMI, are presented in Table 3. Pre-obese and obese subjects (BMI $27.0-40.0 \mathrm{~kg} / \mathrm{m}^{2}$ ) showed higher percentage of fat mass, serum leptin and CRP. The proportion of females in groups decreased as BMI increased.

To investigate whether serum FFA levels were associated to obesity, we determined total $(\mathrm{mM})$ and individual $(\mu \mathrm{g} / \mathrm{mL})$ fasting circulating FFA levels. Our data showed a serum FFA profile linked to obesity that was characterized by decreased levels in eicosapentaenoic acid (EPA) (n-3 series), together with higher levels of linoleic, gamma-linolenic (n-
6 series) and palmitic acids. Total serum FFA showed a slight increase in the obese group compared to normal weight subjects, that did not reach statistical significance $(p=.058)$ (Table 4).

In addition, after adjusting by gender, results from a lineal regression analysis showed that total FFA were directly associated to other obesity related factors such as body fat percentage and MDA. In line with this, linoleic acid was also directly associated with MDA, whereas gamma-linolenic acid was related to CRP (Table 5).

In order to elucidate the role of fecal microbiota and individual serum FFA in obesity, a classification tree was modeled. Fig. 1 shows the model provided by C5.0 for predicting obesity based on BMI classification using fecal microbial groups, serum individual FFA, smoking habit, age and gender. At the top level of the tree, serum EPA concentration was the variable most strongly associated with BMI. Splitting the sample according to EPA concentration in serum, $\leq 0.235 v s$. $>0.235 \mu \mathrm{g} / \mathrm{mL}$, produced two subgroups. In the "high EPA" group the covariate most strongly associated with BMI was the fecal levels of Bacteroides group, which defined subgroups according to whether individuals had $\leq 9.055$ or $>9.055$ ( $\log n^{\circ}$ cells / $g$ of feces). In this sense, subjects with higher serum concentration of EPA and higher fecal levels of Bacteroides belonged to normal weight group, while subjects showing lower counts of Bacteroides group were overweight. These results point out to the increased concentrations of EPA as the main non-obese linked factor.

Within the second group (following the left branch in Fig. 1), with "low EPA" serum levels, none of the covariates were directly associated with the outcome. In this classification, the strongest association was with fecal levels of Faecalibacterium $\leq 6.456$ or $>6.456\left(\log \mathrm{n}^{\circ}\right.$ cells $/ \mathrm{g}$

Table 3

General characteristics of the study population according to Body Mass Index $\left(\mathrm{kg} / \mathrm{m}^{2}\right)$.

\begin{tabular}{|c|c|c|c|c|c|}
\hline & Normal weight & Overweight & $\mathrm{p}$ & Pre- Obesity/Obesity & $\mathrm{p}$ \\
\hline & $<25.0 \mathrm{~kg} / \mathrm{m}^{2}$ & $25.0-26.9 \mathrm{~kg} / \mathrm{m}^{2}$ & & $\geq 27.0 \mathrm{~kg} / \mathrm{m}^{2}$ & \\
\hline & $\mathrm{N}=20$ & $\mathrm{~N}=12$ & & $\mathrm{~N}=34$ & \\
\hline Age (years) ${ }^{\mathrm{a}}$ & $56 \pm 10.1$ & $55.5 \pm 8.3$ & 0.501 & $49.6 \pm 12.0$ & 0.129 \\
\hline Female $(\%)^{\mathrm{b}}$ & 80.0 & 75.0 & 0.740 & 44.1 & 0.010 \\
\hline Body fat $(\%)^{\mathrm{b}}$ & $29.0 \pm 7.4$ & $36.2 \pm 9.6$ & 0.016 & $38.9 \pm 12.6$ & 0.015 \\
\hline Energy intake $(\mathrm{kcal} / \mathrm{d})^{\mathrm{a}}$ & $1936.0 \pm 537.2$ & $1790.7 \pm 642.9$ & 0.239 & $1937.2 \pm 456.6$ & 0.802 \\
\hline Alcohol consumers $(\%)^{\mathrm{b}}$ & 70.0 & 50.0 & 0.258 & 58.8 & 0.411 \\
\hline Current smoker (\%) & 27.8 & 18.2 & 0.835 & 27.3 & 0.672 \\
\hline Sedentary lifestyle (\%) ${ }^{\mathrm{b}}$ & 35.0 & 50.0 & 0.403 & 55.9 & 0.138 \\
\hline \multicolumn{6}{|l|}{ Blood parameters ${ }^{\mathrm{a}}$} \\
\hline Leptin (ng/mL) & $6.9 \pm 4.3$ & $9.1 \pm 5.0$ & 0.195 & $10.5 \pm 6.4$ & 0.035 \\
\hline $\operatorname{MDA}(\mu \mathrm{M})$ & $2.2 \pm 0.6$ & $2.1 \pm 0.6$ & 0.744 & $2.5 \pm 1.3$ & 0.542 \\
\hline $\mathrm{CRP}(\mathrm{mg} / \mathrm{L})$ & $0.8 \pm 0.8$ & $1.6 \pm 1.6$ & 0.083 & $5.3 \pm 9.4$ & 0.004 \\
\hline
\end{tabular}

a Results from U-Mann Whitney analysis were presented as mean \pm standard deviation.

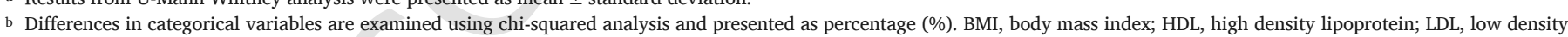
lipoprotein; MDA, malondialdehyde; CRP, C-reactive protein. $p$ value was calculated using normal weight as reference.

Table 4

Differences in main total $(\mathrm{mM})$ and individual serum FFA $(\mu \mathrm{g} / \mathrm{mL})$ according to BMI $\left(\mathrm{kg} / \mathrm{m}^{2}\right)$.

\begin{tabular}{|c|c|c|c|c|c|}
\hline & Normal weight & Over weight & $\mathrm{p}$ & Pre- Obesity/Obesity & $\mathrm{p}$ \\
\hline & $<25.0$ & $25.0-26.9$ & & $\geq 27.0$ & \\
\hline & $\mathrm{N}=20$ & $\mathrm{~N}=12$ & & $\mathrm{~N}=34$ & \\
\hline Total FFA & $0.28 \pm 0.114$ & $0.29 \pm 0.193$ & 1.000 & $0.39 \pm 0.195$ & 0.058 \\
\hline Arachidonic acid & $2.04 \pm 1.01$ & $1.86 \pm 1.15$ & 0.954 & $2.30 \pm 1.65$ & 0.872 \\
\hline Docosahexaenoic acid (DHA) & $1.94 \pm 1.35$ & $1.89 \pm 1.91$ & 0.526 & $1.48 \pm 1.99$ & 0.079 \\
\hline Eicosapentaenoic acid (EPA) & $0.26 \pm 0.40$ & $0.22 \pm 0.19$ & 0.924 & $0.10 \pm 0.13$ & 0.012 \\
\hline Stearic acid & $29.53 \pm 7.42$ & $30.38 \pm 6.18$ & 0.774 & $29.13 \pm 7.76$ & 0.872 \\
\hline Linoleic acid & $7.58 \pm 3.24$ & $9.95 \pm 5.94$ & 0.477 & $12.68 \pm 9.16$ & 0.024 \\
\hline Linolenic acid & $0.22 \pm 0.11$ & $0.26 \pm 0.16$ & 0.477 & $0.27 \pm 0.25$ & 0.809 \\
\hline Oleic acid & $26.97 \pm 12.81$ & $29.42 \pm 15.97$ & 0.659 & $33.45 \pm 19.92$ & 0.282 \\
\hline Palmitoleic acid & $0.80 \pm 0.78$ & $2.10 \pm 2.75$ & 0.326 & $1.24 \pm 1.39$ & 0.136 \\
\hline Gamma-linolenic acid & $0.07 \pm 0.04$ & $0.10 \pm 0.07$ & 0.239 & $1.11 \pm 0.08$ & 0.013 \\
\hline Palmitic acid & $26.49 \pm 6.01$ & $28.9 \pm 9.41$ & 0.552 & $31.47 \pm 8.74$ & 0.038 \\
\hline
\end{tabular}


Table 5

Association between total $(\mathrm{mM})$ and individual $(\mu \mathrm{g} / \mathrm{mL})$ serum FFA characterized in obesity, with obesity related factors.

\begin{tabular}{|c|c|c|c|c|c|c|c|c|c|c|}
\hline & \multicolumn{2}{|c|}{ BMI $\left(\mathrm{kg} / \mathrm{m}^{2}\right)$} & \multicolumn{2}{|c|}{$\%$ Body Fat } & \multicolumn{2}{|c|}{ Leptin (ng/mL) } & \multicolumn{2}{|c|}{$\operatorname{MDA}(\mu \mathrm{M})$} & \multicolumn{2}{|c|}{ CRP (mg/L) } \\
\hline & $R^{2}$ & $\beta$ & $R^{2}$ & $\beta$ & $R^{2}$ & $\beta$ & $R^{2}$ & $\beta$ & $R^{2}$ & $\beta$ \\
\hline Total FFA & 0.122 & 0.191 & 0.270 & $0.237^{*}$ & 0.091 & -0.094 & 0.185 & $0.397 *$ & 0.013 & 0.085 \\
\hline Eicosapentaenoic acid (EPA) & 0.316 & -0.216 & 0.238 & -0.133 & 0.123 & -0.201 & 0.063 & -0.132 & 0.042 & -0.188 \\
\hline Linoleic acid & 0.105 & 0.129 & 0.239 & 0.141 & 0.102 & -0.143 & 0.140 & $0.320 *$ & 0.045 & 0.206 \\
\hline Gamma-linolenic acid & 0.128 & 0.198 & 0.249 & 0.172 & 0.113 & 0.175 & 0.092 & 0.218 & 0.100 & $0.309^{*}$ \\
\hline Palmitic acid & 0.102 & 0.112 & 0.233 & 0.116 & 0.094 & -0.109 & 0.096 & 0.230 & 0.009 & 0.050 \\
\hline
\end{tabular}

Results from lineal regression analysis adjusted by gender. FFA, free fatty acids; MDA, malondialdehyde; CRP, C-reactive protein; ${ }^{*} p$ value $\leq .05$.

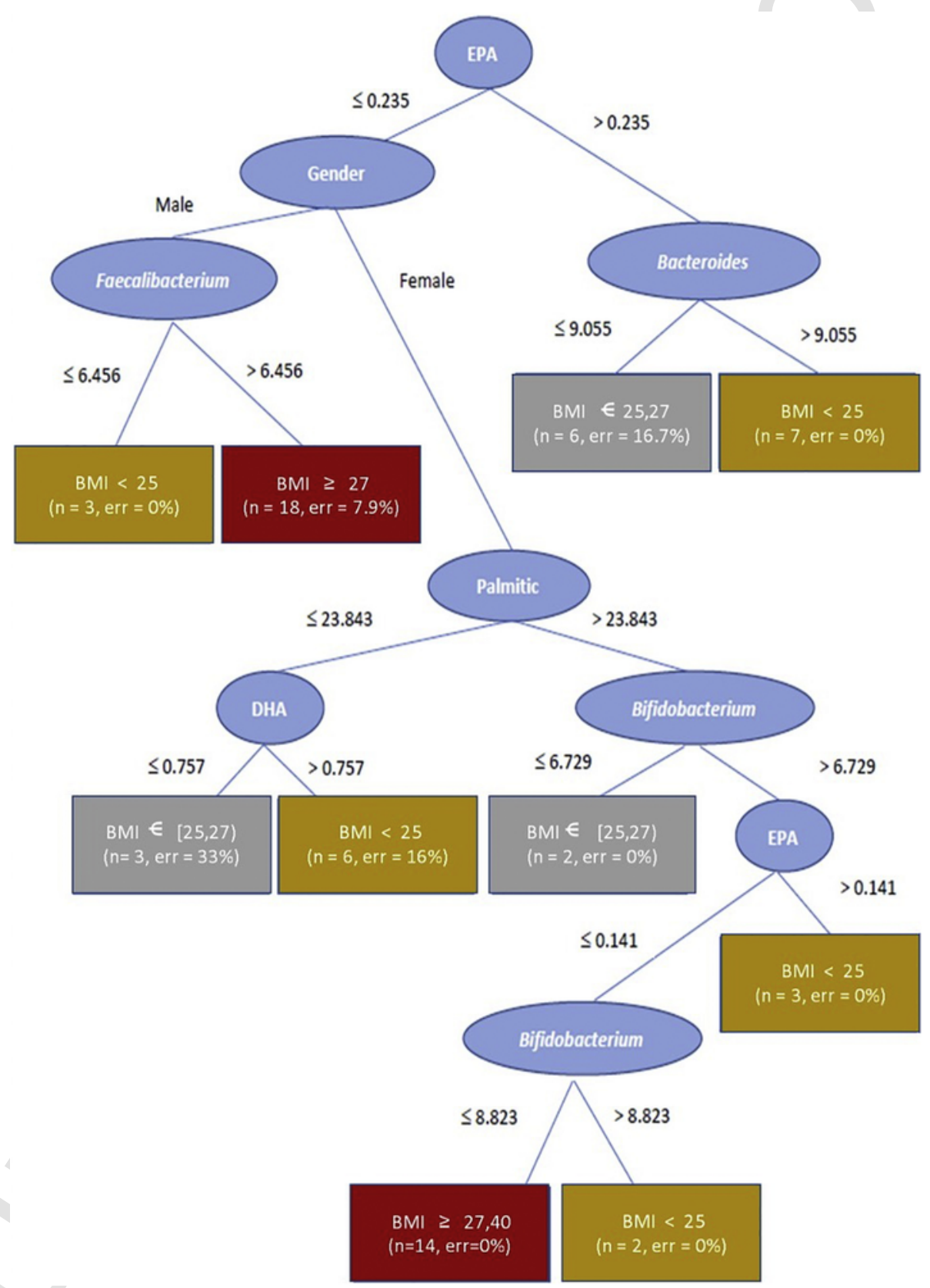

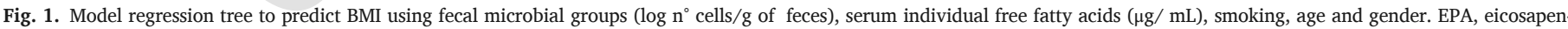
taenoic acid; DHA, docosahexaenoic acid; n, number of subjects; err, error rate; $\epsilon$, includes.

of feces) which was associated in the male group to normal and pre-obesity/obesity status, respectively.

Furthermore, for female, within the "low EPA" group the covariate most strongly associated with obesity was palmitic acid, which defines subgroups according to whether individuals presented serum levels $\leq 23.843$ or $>23.843 \mu \mathrm{g} / \mathrm{mL}$; so, those women who presented lower concentration of palmitic acid $(\leq 23.843 \mu \mathrm{g} / \mathrm{mL})$ together with docosahexaenoic acid (DHA) concentrations $>0.757 \mu \mathrm{g} / \mathrm{mL}$ were classified as 
normal weight. On the other hand, when palmitic acid was $>23.843 \mu \mathrm{g} /$ $\mathrm{mL}$ and fecal levels of Bifidobacterium showed counts $\leq 6.729\left(\log \mathrm{n}^{\circ}\right.$ cells / $\mathrm{g}$ of feces), females were classified within the overweight group. In the same way, when following the right branch, the individuals with "high Bifidobacterium" levels were further divided on the basis of EPA concentration $(\leq 0.141$ or $>0.141 \mu \mathrm{g} / \mathrm{mL}$ ). In this scenario, if EPA was $>0.141 \mu \mathrm{g} / \mathrm{mL}$, or if it was $\leq 0.141 \mu \mathrm{g} / \mathrm{mL}$, running together with Bifidobacterium $>8.823$ ( $\log \mathrm{n}^{\circ}$ cells $/ \mathrm{g}$ of feces), subjects were labeled as normal weight. Overall, these data suggest serum EPA as a significant obesity indicator with independence of the rest of the variables. Furthermore, when the concentration of serum EPA is $\leq 0.235 \mu \mathrm{g} / \mathrm{mL}$, the interaction between serum FFA and the gut microbiota seems to be sex-dependent, being associated to Faecalibacterium and Bifidobacterium for males and females, respectively.

One of the main characteristics of some classification trees, and in particular those obtained by C5.0 algorithm, is that they are able to select those relevant features affecting the variable to predict. Thus, it is possible to estimate the role of fecal microbiota in the observed associations. According to that, the importance of microbial groups and serum FFA to classify our sample according to BMI is shown in Supplementary Fig. 1. Serum EPA and gender were identified as the most significant variables with $100 \%$ and $80 \%$ of importance, respectively. Palmitic acid, Bifidobacterium and Faecalibacterium explained $>30 \%$, followed by Bacteroides group with 20\% and DHA almost with $15 \%$ of importance.

Given the relevant role of serum EPA as an obesity indicator, daily consumption of major food groups was compared according to EPA cut-off point. Subjects with serum concentrations $>0.235 \mu \mathrm{g} / \mathrm{mL}$, presented higher intake of fish and seafoods, and vegetables (Fig. 2A), specifically from salmon, sardine and tuna, and onion, cauliflower, spinach, green beans and lettuce, respectively (Fig. 2B).

\section{Discussion}

The present work represents a preliminary study to investigate the potential relationship between serum FFA as indicator of dietary fat and the gut microbiota. Identification of novel lipid predictors raises the possibility to improve our understanding of the obesity and associated metabolic changes.

We have identified an obese FFA profile, characterized by the simultaneous reduction of EPA serum levels and increased concentration of linoleic, gamma-linolenic and palmitic acids, as regards of values found in the normal-weight population. In accordance with previous studies carried out in healthy subjects, where the infusion of lipids induced a rise in the concentrations of serum FFA and free radicals (Paolisso et al., 1996), we observed a direct association between total

A)

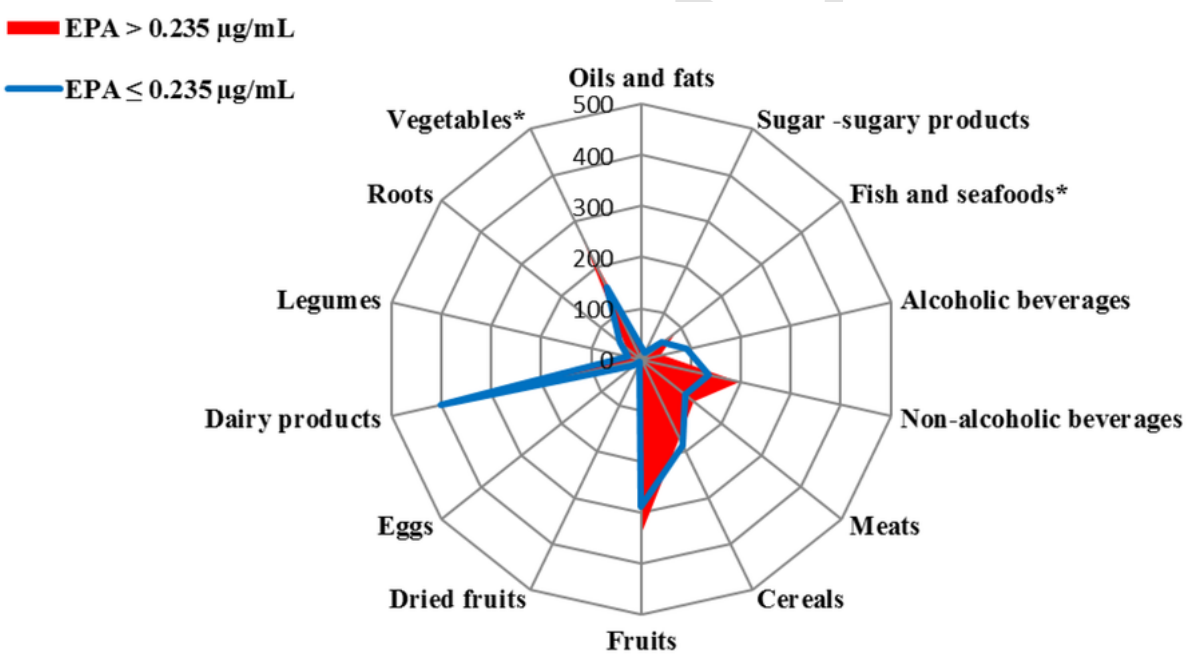

B1)

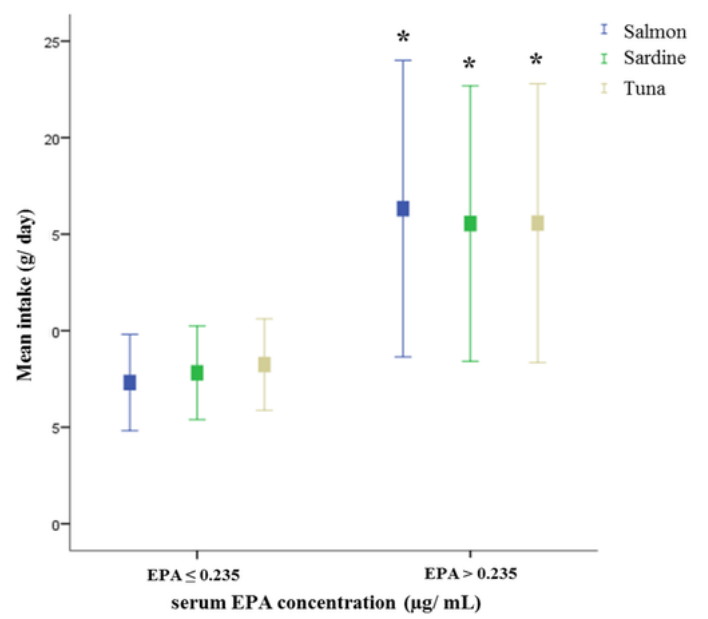

B2)

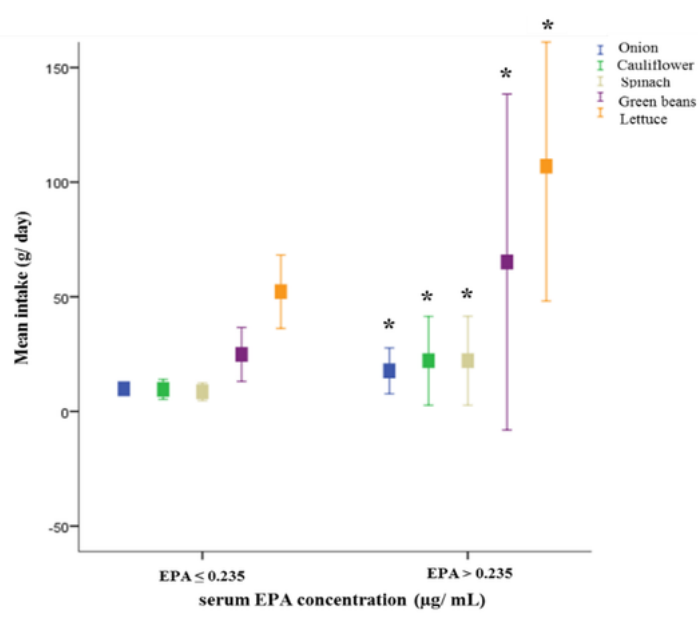

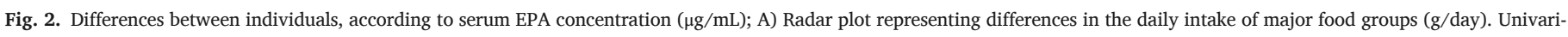

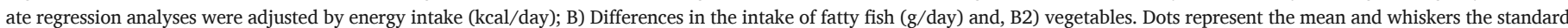

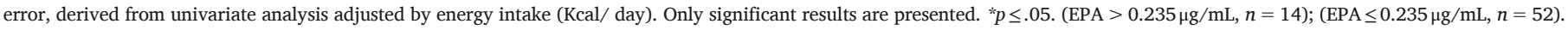


serum FFA and the serum concentration of MDA, suggesting the potential usefulness of total serum FFA as a reliable marker of a microenvironment sensitive to peroxidation (Furukawa et al., 2004). Also, the relationship between serum FFA concentration and obesity, seems to be complex and closely linked to an obesity-linked FFA profile and to subject's gut microbial composition, which has been repeatedly reported to be different in obese humans as compared to lean people, both in terms of diversity and in the relative abundance of the dominant phyla Bacteroidetes and Firmicutes. We observed that gamma-linolenic acid, a precursor of arachidonic acid, was directly related with the concentration of CRP (Paolisso et al., 1996). Despite that our data are in agreement with the notion that total serum FFA concentrations differed according to the grade of obesity and proportion of body fat mass (Karpe et al., 2011), we have only found a modest increase $(0.107 \mathrm{mM})$ in serum total FFA in our pre-obese/obese subjects with respect to the normal weight ones. Therefore, we have analyzed our data considering the well-known differences present in the body composition between male and female, and its role on FFA kinetics; with this in mind, statistical analyses were adjusted by gender to avoid the effect of the said variable as confounder (Alsharari et al., 2017; Blaak, 2001).

Then, taking into consideration that differences in fatty acids can be related to differences in the composition and functionality of the intestinal microbiota, it is possible that these compounds could act as mediators for the development of obesity (Karlsson et al., 2013; Khan et al., 2014; Le Chatelier et al., 2013; Tremaroli \& Bäckhed, 2012; Zhao, 2013) and proinflammatory status frequently associated with it (Boden, 2008; Perreault et al., 2014; Serhan, Chiang, \& Van Dyke, 2008). In the present study, we wanted to go a step further by addressing the potential connections between the gut microbiota and the different serum FFA in the context of obesity, by analyzing in detail the possible interactions between all factors involved. With this purpose, we have constructed a decision tree using the C5.0 machine learning method in order to explore potentially non-linear relationships among different types of FFA, measured in serum, and the main gut representative microbial groups, with the prevalence of obesity as outcome (Venkatasubramaniam et al., 2017). This approach could offer up a better picture of what is occurring in a physiological context than the traditional analyses which are conducted to decipher the effect of individual variables; this approach has also the additional advantage of offering a cut-off point that could be useful for classifying the risk of obesity of the subjects evaluated. Our results using this method, pointed to serum EPA concentration, running together with fecal levels of Bacteroides group, as the best predictor of BMI, with independence of gender and the rest of the variables introduced in the model. In this regard, reduced intestinal Bacteroides levels were repeatedly reported in obese subjects (Duca et al., 2014; Liu et al., 2017; Park et al., 2015) and some studies have also shown enrichment in this bacterial group after weight loss (Jumpertz et al., 2011) or after bariatric surgery (Furet et al., 2010). Moreover, high concentrations of omega-3 fatty acids in blood have been associated with protection against obesity and obesity-related clinical manifestations (Huang et al., 2016; Scaglioni et al., 2006). Omega-3 fatty acids could be related with anti-inflammatory effects, and dietary supplementation with PUFA-Omega 3 seems to be associated with some common changes in the gut microbiota. Particularly, these changes mostly focused to a decrease in Faecalibacterium (Costantini, Molinari, Farinon, \& Merendino, 2017), often associated to an increase of the phylum Bacteroidetes and of some butyrate-producing bacteria from the Lachnospiraceae family, included in Clostridium cluster XIVa, particularly those from the genus Roseburia. Moreover, PUFA have been recently associated with the pathogenesis of obesity, with the imbalance between n-6 and n-3 PUFA playing a role in the increase of body weight (Albracht-Schulte et al., 2018; Costantini et al., 2017; Huang et al., 2016). However, despite the extensive research already available relating dietary fatty acids, serum FFA and intestinal microbiota composition and function with obesity, this preliminary study represents the first attempt to establish cut-off points for the intestinal microbiota and serum FFA as obesity predictors, corroborating the importance of considering together intestinal microbiota and serum FFA in the regulation of body weight. Based on the results of the decision tree generated with the algorithm C5.0, we propose that serum EPA concentrations above $0.235 \mu \mathrm{g} / \mathrm{mL}$ and levels of Bacteroides higher than $9.055 \log n^{\circ}$ cells per $g$ of feces, occurring simultaneously in the same individual, could be considered as a predictor of normal weight independently of gender. Although the influence of gender in human gut microbiota remains unclear, some authors have reported a lower abundance of the genera Bacteroides in men than in women for BMI above $33 \mathrm{~kg} / \mathrm{m}^{2}$ (Haro et al., 2016). In this sense, the absence of gender-specific differences in our sample were expectable since Bacteroides only stratifies subjects with normal weight or overweight (in both cases with BMI $<27 \mathrm{~kg} / \mathrm{m}^{2}$ ). Furthermore, according to the well-known evidence about a sex-specific regulation of lipid metabolism, we have found that Faecalibacterium and Bifidobacterium might play an important role by complementing the levels of FFA for males and females, respectively. The different bacterial populations assessed in this study (Akkermansia, Bacteroides group, Bifidobacterium, Clostridia cluster XIVa group, Lactobacillus group and Faecalibacterium prausnitzii) represent $>90 \%$ of the overall phylogenetic types of the human intestinal microbiota (Yatsunenko et al., 2012).

Pieces of evidence illustrating the role of $F$. prausnitzii in the maintenance of intestinal health have been mainly focused to the anti-inflammatory effect of this microorganism, suggesting a possible benefit against the development of IBD (inflammatory bowel disease) or Crohn's diseases (Cao, Shen, \& Ran, 2014). However, these abundant anaerobic bacteria belonging to the phylum Firmicutes (Cao et al., 2014) could also play a differential role in obesity. It has been recently suggested that this butyrate producer is capable of improving the gut barrier function and is altered in pathologies characterized by low grade inflammation like obesity (Stenman, Burcelin, \& Lahtinen, 2016).

Given the observational nature of our study, we cannot conclude any causal relationships or directionality between the above-mentioned variables. Indeed, the causal relationship between the gut microbiota and several pathologies including obesity is still unclear and long-term dietary habits have been reported to create inter-individual variation in microbiota composition (Flint, Duncan, \& Louis, 2017). In addition, the role of FFA in obesity has not still been experimentally proven and some of our results may be influenced by men/women ratio. Nevertheless, if we consider that diets with high content in fat or in long chain fatty acids were found to modulate the gut microbiome by promoting obesity (Alcock \& Lin, 2015) while, dietary n-3 polyunsaturated fatty acids have been shown to protect against dysbiosis (Alcock \& Lin, 2015), it is tempting to speculate with the possibility of modifying the obese-linked FFA profile and the altered gut microbiota through dietary interventions attending to gender differences.

In this sense, as we expected, we have evidenced that subjects with serum EPA concentration above the cut-off point $(0.235 \mu \mathrm{g} / \mathrm{mL})$, showed higher intake of fish and seafoods than the rest of the sample (53.02 vs. $88.88 \mathrm{~g} / \mathrm{d}$ ). These differences in the amount of fish consumed, were explained by a higher intake of rich-n-3 PUFAs fatty fish as salmon, sardine and tuna, suggesting a direct correlation between the EPA serum concentration and its daily intake.

\section{Conclusions}

These results reveal the different pattern of serum fatty acids as potential predictors of obesity, supporting the pivotal role of the gut microbiota in this complex interrelationship. The underlying mechanisms explaining these associations should be investigated in future intervention studies that could provide new hypotheses to reduce the incidence of obesity and to develop optimal strategies for early prevention and detection of related disorders. 


\section{Uncited references}

Cavalcante-Silva et al., 2015

Cuervo et al., 2015

Dao and Clément, 2018

Flint et al., 2017

Centro de Enseñanza Superior de Nutrición Humana y Dietética, CESNID, 2008

Park et al., 2013

\section{Acknowledgments}

We show our greatest gratitude to all the volunteers participating in the study.

\section{Funding}

This work was funded through the project GRUPIN14-043 "Microbiota Humana, Alimentación y Salud" granted by "Plan Regional de Investigación del Principado de Asturias", Spain, and by the Alimerka Foundation. NS is the recipient of a postdoctoral contract awarded by the Fundación para la Investigación Biosanitaria de Asturias (FINBA). Public National and Regional grants received cofounding from European Union FEDER funds.

\section{Author contributions}

S. González had the primary responsibility in the study design and protocol development and confirms that she had full access to the data in the study and final responsibility for the decision to submit for publication. Together with T. Fernández-Navarro they contributed to data analysis and writing of the manuscript. I. Díaz performed and developed the machine learning algorithm and contributed to the writing of the paper. I. Gutiérrez-Díaz was involved in data collection and performed data and statistical. J- Rodríguez-Carrio and A. Suárez have determined the serum free fatty acids and data processing. C. G. de los Reyes-Gavilán, M. Gueimonde and N. Salazar have conducted the microbial analysis and data processing and have supervised the design and execution of the study and data analysis and contributed to the writing of the paper. All authors critically reviewed the manuscript and approved the final version submitted for publication.

\section{Declarations of interest}

None.

\section{Appendix A. Supplementary data}

Supplementary data to this article can be found online at https:// doi.org/10.1016/j.foodres.2018.12.009.

\section{References}

Albracht-Schulte, K., Kalupahana, N.S., Ramalingam, L., Wang, S., Rahman, S., Robert-McComb, J., Moustaid-Moussa, N., 2018. Omega-3 fatty acids in obesity and metabolic syndrome: A mechanistic update. The Journal of Nutritional Biochemistry 27, 1-16. https://doi.org/10.1016/j.jnutbio.2018.02.012.

Alcock, J., Lin, H.C., 2015. Fatty acids from diet and microbiota regulate energy metabolism. F1000Research 4 (F1000 Faculty Rev)https://doi.org/10.12688/f1000research. 6078.1 .

Alsharari, Z.D., Risérus, U., Leander, K., Sjögren, P., Carlsson, A.C., Vikström, M., ... Marklund, M., 2017. Serum fatty acids, desaturase activities and abdominal obesity - a population-based study of 60-year old men and women. PLoS One 12 (1)https://doi.org/ 10.1371/journal.pone.0170684.

Arboleya, S., Binetti, A., Salazar, N., Fernandez, N., Solis, G., Hernandez-Barranco, A., .. Gueimonde, M., 2012. Establishment and development of intestinal microbiota in preterm neonates. FEMS Microbiology Ecology 79, 763-772 https://doi https://doi. org/10.1111/j.1574-6941.2011.01261.x.
Blaak, E., 2001. Gender differences in fat metabolism. Current Opinion in Clinical Nutrition and Metabolic Care 4, 499-502. https://doi.org/10.1097/ 00075197-200111000-00006.

Boden, G., 2008. Obesity and free fatty acids. Endocrinology and Metabolism Clinics of North America 37, 635-646. https://doi.org/10.1016/j.ecl.2008.06.007.

Cao, Y., Shen, J., Ran, Z.H., 2014. Association between faecalibacterium prausnitzii reduction and inflammatory bowel disease: A meta-analysis and systematic review of the literature. Gastroenterology Research and Practice 2014, https://doi.org/10.1155/ 2014/872725.

Cavalcante-Silva, L.H.A., Galvão, J.G.F.M., da Silva, J.S., De, F., de Sales-Neto, J.M., Rodrigues-Mascarenhas, S., 2015. Obesity-driven gut microbiota inflammatory pathways to metabolic syndrome. Frontiers in Physiology 6, 341. https://doi.org/10.3389/ fphys.2015.00341.

Clarke, G., Stilling, R.M., Kennedy, P.J., Stanton, C., Cryan, J.F., Dinan, T.G., 2014. Minireview: Gut microbiota: The neglected endocrine organ. Molecular Endocrinology 28 1221 - 38 https://doi.org/10.1210/me.2014-1108.

Costantini, L., Molinari, R., Farinon, B., Merendino, N., 2017. Impact of omega-3 fatty acids on the gut microbiota. International Journal of Molecular Sciences 18, 2645. https://doi.org/10.3390/ijms18122645.

Cuervo, A., Hevia, A., Lopez, P., Suarez, A., Sanchez, B., Margolles, A., Gonzalez, S., 2015 Association of polyphenols from oranges and apples with specific intestinal microorganisms in systemic lupus erythematosus patients. Nutrients 7, 1301-1317. https:// doi.org/10.3390/nu7021301.

Cuervo, A., Valdes, L., Salazar, N., Gd.l.R.-G., Ruas-Madiedo, P., Gueimonde, M., Gonzalez, S., 2014. Pilot study of diet and microbiota: Interactive associations of fibers and polyphenols with human intestinal bacteria. Journal of Agricultural and Food Chemistry 62, https://doi.org/10.1021/jf501546a, 5330 - 6 .

Dao, M.C., Clément, K., 2018. Gut microbiota and obesity: Concepts relevant to clinical care. European Journal of Internal Medicine 48, 18-24. https://doi.org/10.1016/j. ejim.2017.10.005.

Dao, M.C., Everard, A., Aron-Wisnewsky, J., Sokolovska, N., Prifti, E., Verger, E.O., Lepage, P., 2016. Akkermansia muciniphila and improved metabolic health during a dietary intervention in obesity: Relationship with gut microbiome richness and ecology. Gut 65, 426-436. https://doi.org/10.1136/gutjnl-2014-308778.

De los Reyes-Gavilán, C.G., Delzenne, N.M., González, S., Gueimonde, M., Salazar, N., 2014. Development of functional foods to fight against obesity - Opportunities for probiotics and prebiotics. Agro Food Industry Hi-Tech 25, 35-39 https://hdl.handle.net/ 2078.1/172693.

Demarco, V.G., Aroor, A.R., Sowers, J.R., 2014. The pathophysiology of hypertension in patients with obesity. Nature Reviews. Endocrinology 10, 364-376. https://doi.org/ 10.1038/nrendo.2014.44

Duca, F.A., Sakar, Y., Lepage, P., Devime, F., Langelier, B., Dore, J., Covasa, M., 2014. Replication of obesity and associated signaling pathways through transfer of microbiota from obese-prone rats. Diabetes 63, 1624-1636. https://doi.org/10.2337/ db13-1526.

Emanuela, F., Grazia, M., Marco, D.R., Maria Paola, L., Giorgio, F., Marco, B., 2012. Inflammation as a link between obesity and metabolic syndrome. Journal of Nutrition and Metabolism. https://doi.org/10.1155/2012/476380.

Feng, J., Tang, H., Li, M., Pang, X., Wang, L., Zhang, M., ... Shen, J., 2014. The abundance of fecal Faecalibacterium prausnitzii in relation to obesity and gender in Chinese adults. Arch Microbiol 196, 73-77. https://doi.org/10.1007/s00203-013-0942-2.

Flint, H.J., Duncan, S.H., Louis, P., 2017. The impact of nutrition on intestinal bacterial communities. Current Opinion in Microbiology 38, 59-65. https://doi.org/10.1016/j. mib.2017.04.005.

Furet, J.P., Kong, L.C., Tap, J., Poitou, C., Basdevant, A., Bouillot, J.L., Clément, K. 2010. Differential adaptation of human gut microbiota to bariatric surgery-induced weight loss: Links with metabolic and low-grade inflammation markers. Diabetes 59, 3049-3057. https://doi.org/10.2337/db10-0253.

Furukawa, S., Fujita, T., Shimabukuro, M., Iwaki, M., Yamada, Y., Nakajima, Y., Shimomura, I., 2004. Increased oxidative stress in obesity and its impact on metabolic syndrome. The Journal of Clinical Investigation 114, 1752-1761. https://doi.org/10. $1172 / \mathrm{JCI} 21625$.

Gerard-Monnier, D., Erdelmeier, I., Regnard, K., Moze-Henry, N., Yadan, J.C., Chaudiere J., 1998. Reactions of 1-methyl-2-phenylindole with malondialdehyde and 4-hydroxyalkenals. Analytical applications to a colorimetric assay of lipid peroxidation. Chemical Research in Toxicology 11, 1176-1183. https://doi.org/10.1021/tx9701790.

Haro, C., Rangel-Zúñiga, O.A., Alcalá-Díaz, J.F., Gómez-Delgado, F., Pérez-Martínez, P., Delgado-Lista, J., ... Camargo, A., 2016. Intestinal microbiota is influenced by gender and body mass index. PLoS One 11, e0154090https://doi.org/10.1371/journal.pone. 0154090.

Huang, C.W., Chien, Y.S., Chen, Y.J., Ajuwon, K.M., Mersmann, H.M., Ding, S.T., 2016 Role of n-3 polyunsaturated fatty acids in ameliorating the obesity-induced metabolic syndrome in animal models and humans. International Journal of Molecular Sciences 17, https://doi.org/10.3390/ijms17101689.

Centro de Enseñanza Superior de Nutrición Humana y Dietética, CESNID, 2008. Tablas de Composición de Alimentos por Medidas Caseras de Consumo Habitual en España. Barcelona, Spain.

Jumpertz, R., Le, D.S., Turnbaugh, P.J., Trinidad, C., Bogardus, C., Gordon, J.I., Krakoff, J., 2011. Energy-balance studies reveal associations between gut microbes, caloric load, and nutrient absorption in humans. The American Journal of Clinical Nutrition 94 58-65. https://doi.org/10.3945/ajcn.110.010132.

Karlsson, F.H., Tremaroli, V., Nookaew, I., Bergström, G., Behre, C.J., Fagerberg, B., Bäckhed, F., 2013. Gut metagenome in European women with normal, impaired and diabetic glucose control. Nature 498, 99-103. https://doi.org/10.1038/nature12198.

Karpe, F., Dickmann, J.R., Frayn, K.N., 2011. Fatty acids, obesity, and insulin resistance: Time for a reevaluation. Diabetes 60, 2441-2449. https://doi.org/10.2337/ db11-0425.

Khan, M.T., Nieuwdorp, M., Bäckhed, F., 2014. Microbial modulation of insulin sensitivity. Cell Metabolism 20, 753-760. https://doi.org/10.1016/j.cmet.2014.07.006. 
T. Fernández-Navarro et al.

Food Research International $x x x$ (2018) $x x x-x x x$

Kuhn, M.Johnson, K., 2016. Applied predictive modeling. Springer, New York.

Le Chatelier, E., Nielsen, T., Qin, J., Prifti, E., Hildebrand, F., Falony, G., Yamada, T., 2013. Richness of human gut microbiome correlates with metabolic markers. Nature 500, 541-546. https://doi.org/10.1038/nature12506.

Lee, J.S., Pinnamaneni, S.K., Eo, S.J., Cho, I.H., Pyo, J.H., Kim, C.K., Watt, M.J., 2006. Saturated, but not n-6 polyunsaturated, fatty acids induce insulin resistance: Role of intramuscular accumulation of lipid metabolites. Journal of Applied Physiology (Bethesda, Md.: 1985) 100, 1467-1474. https://doi.org/10.1152/japplphysiol.01438. 2005.

Liu, R., Hong, J., Xu, X., Feng, Q., Zhang, D., Gu, Y., Wang, W., 2017. Gut microbiome and serum metabolome alterations in obesity and after weight-loss intervention. Nature Medicine 23, 859-868. https://doi.org/10.1038/nm.4358.

Million, M., Maraninchi, M., Henry, M., Armougom, F., Richet, H., Carrieri, P., ... Raoult, D., 2012. Obesity-associated gut microbiota is enriched in Lactobacillus reuteri and depleted in Bifidobacterium animalis and Methanobrevibacter smithii. International Journal of Obesity 36, 817-825. https://doi.org/10.1038/ijo.2011.153.

Paolisso, G., Gambardella, A., Tagliamonte, M.R., Saccomanno, F., Salvatore, T., Gualdiero, P., Howard, B.V., 1996. Does free fatty acid infusion impair insulin action also through an increase in oxidative stress. The Journal of Clinical Endocrinology and Metabolism 81, 4244-4248. https://doi.org/10.1210/jc.81.12.4244.

Park, J.S., Seo, J.H., Youn, H.S., 2013. Gut microbiota and clinical disease: Obesity and nonalcoholic fatty liver disease. Pediatric Gastroenterology, Hepatology and Nutrition 16, 22-27. https://doi.org/10.5223/pghn.2013.16.1.22.

Perreault, M., Roke, K., Badawi, A., Nielsen, D.E., Abdelmagid, S.A., El-Sohemy, A., Mutch, D.M., 2014. Plasma levels of 14:0, 16:0, 16:1n-7, and 20:3n-6 are positively associated, but 18:0 and 18:2n-6 are inversely associated with markers of inflammation in young healthy adults. Lipids 49, 255-263. https://doi.org/10.1007/ s11745-013-3874-3.

Pizarro, C., Arenzana-Rámila, I., Pérez-Del-Notario, N., Pérez-Matute, P., González-Sáiz, J.M., 2013. Plasma lipidomic profiling method based on ultrasound extraction and liquid chromatography mass spectrometry. Analytical Chemistry 85, 12085-12092. https://doi.org/10.1021/ac403181c.

Powers, M.W., 2011. Evaluation: From precision, recall and F-measure to ROC, informedness and correlation. Journal of Machine Learning Technologies 2, 37-63.
Rodríguez-Carrio, J., Salazar, N., Margolles, A., González, S., Gueimonde, M., de los Reyes-Gavilán, C.G., Suárez, A., 2017. Free fatty acids profiles are related to gut microbiota signatures and short-chain fatty acids. Frontiers in Immunology 8, https:// doi.org/10.3389/fimmu.2017.00823.

Salas-Salvado, J., Rubio, M.A., Barbany, M., Moreno, B., 2007. SEEDO 2007, Consensus for the evaluation of overweight and obesity and the establishment of therapeutic intervention criteria. Medicina Clínica 128, 184-196.

Scaglioni, S., Verduci, E., Salvioni, M., Bruzzese, M.G., Radaelli, G., Zetterström, R., Agostoni, C., 2006. Plasma long-chain fatty acids and the degree of obesity in Italian children. Acta Paediatrica International Journal of Paediatrics 95, 964-969. https://doi. org/10.1080/08035250600764834.

Serhan, C.N., Chiang, N., Van Dyke, T.E., 2008. Resolving inflammation: Dual anti-inflammatory and pro-resolution lipid mediators. Nature Reviews. Immunology 8, 349-361. https://doi.org/10.1038/nri2294.

Stenman, L.K., Burcelin, R., Lahtinen, S., 2016. Establishing a causal link between gut microbes, body weight gain and glucose metabolism in humans - towards treatment with probiotics. Beneficial Microbes 7, 11-22. https://doi.org/10.3920/BM2015.0069.

Trayhurn, P., 2005. Adipose tissue in obesity - an inflammatory issue. Endocrinology 146, 1003-1005. https://doi.org/10.1210/en.2004-1597.

Tremaroli, V., Bäckhed, F., 2012. Functional interactions between the gut microbiota and host metabolism. Nature 489, 242-249. https://doi.org/10.1038/nature11552.

Venkatasubramaniam, A., Wolfson, J., Mitchell, N., Barnes, T., Jaka, M., French, S., 2017. Decision trees in epidemiological research. Emerging Themes in Epidemiology 14 (11)https://doi.org/10.1186/s12982-017-0064-4

World Health Organization, 2016. WHO|Obesity and overweight. WHO https://doi.org// entity/mediacentre/factsheets/fs311/en/index.html.

Yatsunenko, T., Rey, F.E., Manary, M.J., Trehan, I., Dominguez-Bello, M.G., Contreras, M., Magris, M., Hidalgo, G., Baldassano, R.N., Anokhin, A.P., et al., 2012. Human gut microbiome viewed across age and geography. Nature 486, 222-227. https://doi.org/ 10.1038 /nature11053.

Zhao, L., 2013. The gut microbiota and obesity: From correlation to causality. Nature Reviews. Microbiology 11, 639-647. https://doi.org/10.1038/nrmicro3089.

9 